\title{
Investigating the Role of Politeness in Human-Human Online Tutoring
}

\author{
Jionghao Lin ${ }^{1}$, David Lang ${ }^{2}$, Haoran $\mathrm{Xie}^{3}$, Dragan Gašević ${ }^{1}$, \\ and Guanliang Chen ${ }^{1(\otimes)}$ \\ 1 Monash University, Clayton, Australia \\ \{jionghao.lin, dragan.gasevic, guanliang. chen\}@monash.edu \\ 2 Stanford University, Stanford, CA, USA \\ dnlang86@stanford.edu \\ 3 Lingnan University, New Territories, Hong Kong SAR, China \\ hrxie2@gmail.com
}

\begin{abstract}
This study aims to investigate the role of politeness in onlinetutoring practices by analyzing a large-scale human-human tutorial dialogue dataset. To this end, we employed linguistic theories of politeness to identify the politeness strategies contained in utterances made by tutors and students, and these strategies were further combined to quantify the politeness levels of tutors and students in a tutorial session. The results revealed that tutors had a similar level of politeness at the beginning of all dialogues, while students were more polite at the end if they successfully solved problems.
\end{abstract}

Keywords: Educational dialogue analysis $\cdot$ Politeness strategies

\section{Introduction}

Dialogue-based Intelligent Tutoring Systems (ITS), which are expected to act as professional human tutors to teach and interact with students, have been long desired and investigated [7,9,20,21]. Though being popular, most of the existing dialogue-based ITS are still plagued by their ineffectiveness in providing students with personalized learning experiences [1]. The reasons behind such ineffectiveness are multifaceted, among which the lack of sufficient pedagogical expertise was often blamed by researchers [2,5,8,11-14]. In addition to the existing research, we posit that the inability of acting as polite as human tutors might play as another influential role here, which is widely recognized as an integral part of civil behavior in social communications and has been demonstrated essential in several educational settings [10,15-19,22-25]. Instead of directly equipping existing dialogue-based ITS with the ability to deliver polite conversations with students, we first suggest investigating the importance of politeness in humanhuman online tutoring. Specifically, this paper proposes an approach to measure the politeness level of tutors and students in human-human online tutoring. 
Formally, our work was guided by the following research question: to what extent are tutors and students acting politely in human-human online tutoring? To answer this question, we relied on a dataset consisting of over $15 \mathrm{~K}$ dialogues collected in the setting of one-on-one online tutoring. Specifically, we identified the politeness strategies used by tutors and students by applying the linguistic theories of politeness developed in [3], i.e., the application of a politeness strategy can be indicated by the use of certain words and phrases (e.g., "thank" and "appreciate" are often used to express gratitude). Then, we defined a metric called UP-Score, which measures the overall politeness level of tutors and students by taking all of the observed politeness strategies into account. Our work contributes to the literature on dialogue-based ITS with the following main findings: (i) overall, both tutors and students acted politely across the whole tutorial process, among which dialogues with successful problem solving displayed a lower level of politeness than those without; (ii) tutors displayed a similar level of politeness at the beginning of all categories of dialogues, while students were more polite at the end if they successfully solved problems.

\section{Approach}

In human-human online tutoring, each tutorial session can be regarded as a series of requests, e.g., students request help from tutors to solve problems and tutors also request students to perform certain actions to solve the problems. As indicated in [6], a request from one person to another is likely to and mainly to give rise to negative politeness strategies, which recognize the friendliness with the other person but assume the expressed content would likely pose imposition on the other person [3]. For instance, the negative strategy Gratitude is an effective one for a requester (i.e., student) to help balance out the burden placed on a tutor (e.g., "I would really appreciate if you could help me."). Therefore, we mainly considered negative politeness strategies here. The embodiment of a politeness strategy can be revealed by the use of certain politeness markers and the positions of these markers. We identified the politeness strategies contained in utterances by employing the politeness strategy classifier constructed by Danescu et al. [6] with Support Vector Machines. In total, we considered a total of 21 politeness strategies in this study, which are explained in detail in [6].

It should be noted that the application of a politeness strategy can incur a sense of both politeness and impoliteness, For instance, when the word "please" is placed at the beginning of an utterance (e.g., "Please do ..."), it often incurs a sense of impoliteness. To measure the politeness level of an utterance, we further distinguished the 21 strategies into polite and impolite according to the empirical evidences shown in [6] and defined the Politeness score of an Utterance (UP-Score for short): UP-Score = \# Polite strategies - \# Impolite strategies. A positive UP-Score implies that the utterance is polite, while a negative value suggests an impolite one. Then, the UP-Scores of utterances generated by tutors or students were aggregated to answer the research question. 


\section{Dataset and Results}

This study used the same tutorial dialogue dataset in [4] for analyses and experiments, which consists of over $15 \mathrm{~K}$ dialogues crafted by tutors and students working together to solve problems covering subjects like mathematics and chemistry. To gain a better understanding of the role played by politeness in human-human online tutoring, we manually labelled the dialogues to one of the following categories based on the progress made by a student towards solving a problem: (i) Gap-clarified, which shows no clue whether the student made any learning progress or not; (ii) Gap-explained, the student identified what the underlying problem or error was but did not identified a correct or full solution; and (iii) Gap-bridged, the student successfully solved the problem or a similar problem. Most of the dialogues were of the category gap-bridged $(57.1 \%)$, followed by gap-clarified $(22.4 \%)$ and then gap-explained (20.5\%).

The average UP-Score of students and tutors across different dialogues are given in Table 1. When considering all of the utterances made by tutors and students across all dialogues (Row 1), the UP-Score is 0.80 , which implies that the tutorial sessions took place in a relatively polite atmosphere. Also, tutors were far more polite than students (1.01 vs. 0.49$)$. When delving into the $U P$ Score values of different dialogues, we observe that, surprisingly, Gap-clarified dialogues, where students achieved the least amount of learning, were the most polite one (0.99), followed by Gap-explained (0.76) and then Gap-bridged (0.74). These results motivated us to further check the UP-Score of the utterances made by tutors and students at the beginning and the end of the dialogues (Fig. 1).

Table 1. The avg. UP-Score of students and tutors across dialogues of different categories. Differences were tested with Mann-Whitney test between any two of the three dialogue categories and are all significant $(p<0.001)$.

\begin{tabular}{l|l|l|l|l}
\hline & All & G-clarified & G-explained & G-bridged \\
\hline 1. Avg. UP-Score (tutor \& student) & $0.80 \pm 0.30$ & $0.99 \pm 0.38$ & $0.76 \pm 0.26$ & $0.74 \pm 0.24$ \\
\hline 2. Avg. UP-Score (tutor) & $1.01 \pm 0.41$ & $1.27 \pm 0.54$ & $1.00 \pm 0.35$ & $0.91 \pm 0.31$ \\
\hline 3. Avg. UP-Score (student) & $0.49 \pm 0.39$ & $0.63 \pm 0.53$ & $0.39 \pm 0.32$ & $0.47 \pm 0.33$ \\
\hline
\end{tabular}

Figure 1(a) shows that tutors of all types of dialogues displayed a similar level of politeness at the beginning, but the tutors in the Gap-clarified sessions became more polite than the others starting from the 7th utterance. By analyzing the frequent strategies adopted by tutors between 7 th and 15 th utterances, we found that the tutors in the Gap-clarified sessions used much less impolite strategies of Direct_Start and Direct_Question than the tutors in the Gap-explained and Gapbridged sessions. This is probably because, in Gap-explained and Gap-bridged sessions, the tutors had successfully identified the difficulties encountered by students after the first few utterances and started to help students solve problems by giving direct opinions or concrete suggestions, and thus a higher usage of 
Direct_Start and Direct_Question. When it comes to the end of the dialogues (Fig. 1 (b)), we notice that the politeness of the tutors in the Gap-clarified and Gap-explained sessions had a sudden increase from about 1.0 to 1.8 at the last utterance, while the politeness level of the tutors in the Gap-bridged sessions stayed relatively stable. This is because the polite strategy Apologizing was often employed by the tutors at the last utterance of Gap-clarified and Gap-explained dialogues to convey their apologies to the students for not being able to help. Lastly, we can observe that the students in Gap-bridged sessions were much more polite than the others at the end of dialogues, which is in line with our commonsense that students were likely to use strategies like Gratitude to express the acknowledgment and appreciation for the help provided by the tutors.
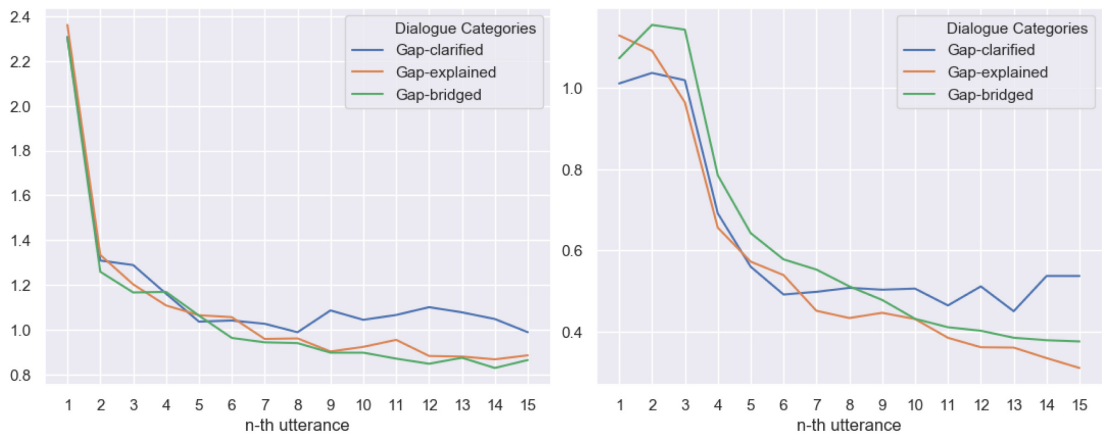

(a) The beginning of tutorial dialogues: tutors (left) and students (right).
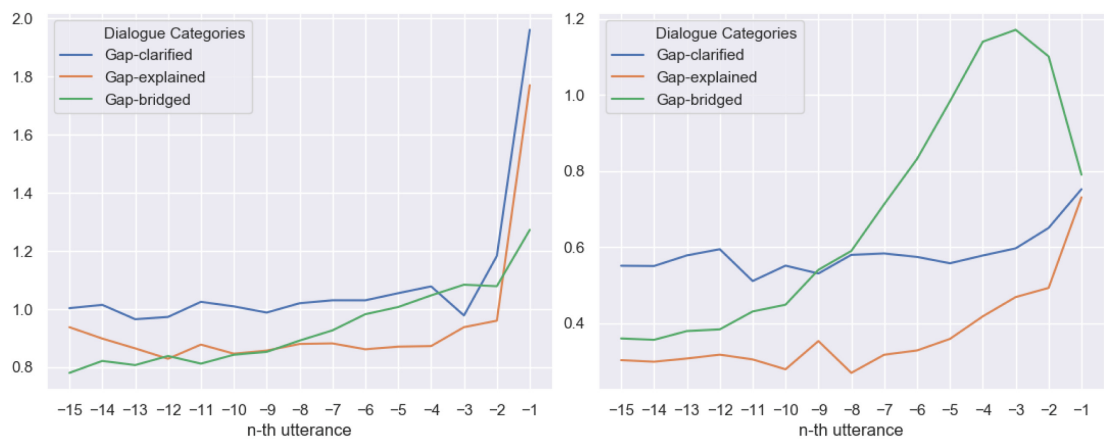

(b) The end of tutorial dialogues: tutors (left) and students (right).

Fig. 1. The avg. UP-Score of tutors/students at the beginning/end of tutorial sessions.

\section{Discussion and Conclusion}

Our study brought several implications for online-tutoring practices. Firstly, though Gap-bridged tutors were likely to be less polite by employing more impolite strategies like Direct_Start, and Direct_Question to guide students after the 
first few utterances, these tutors had helped the students successfully solve problems. This implies that it may be worthy for online tutors to achieve a balance between polite strategies and impolite strategies so as to deliver effective tutoring to students. Secondly, it would be helpful to encourage students to maintain their politeness since the start of a tutorial session, as we observed that students with successful problem solving (i.e., the Gap-bridged ones) were slightly more polite than those without, though the causal relationship between the politeness levels of students and their performance needs to be further verified.

\section{References}

1. Almasri, A., et al.: Intelligent tutoring systems survey for the period 2000-2018 (2019)

2. Boyer, K.E., Phillips, R., Wallis, M.D., Vouk, M.A., Lester, J.C.: Learner characteristics and feedback in tutorial dialogue. In: Proceedings of the Third Workshop on Innovative Use of NLP for Building Educational Applications, pp. 53-61. Association for Computational Linguistics (2008)

3. Brown, P., Levinson, S.: Politeness: Some Universals in Language Usage. Cambridge University Press, Cambridge (1987)

4. Chen, G., Lang, D., Ferreira, R., Gasevic, D.: Predictors of student satisfaction: a large-scale study of human-human online tutorial dialogues. In: EDM (2019)

5. Core, M.G., Moore, J.D., Zinn, C.: The role of initiative in tutorial dialogue. In: EACL (2003)

6. Danescu-Niculescu-Mizil, C., Sudhof, M., Jurafsky, D., Leskovec, J., Potts, C.: A computational approach to politeness with application to social factors. In: Proceedings of the 51st Annual Meeting of the Association for Computational Linguistics (Volume 1: Long Papers), pp. 250-259. Association for Computational Linguistics, Sofia, August 2013. https://www.aclweb.org/anthology/P13-1025

7. Dzikovska, M., Steinhauser, N., Farrow, E., Moore, J., Campbell, G.: Beetle ii: deep natural language understanding and automatic feedback generation for intelligent tutoring in basic electricity and electronics. IJAIED 24(3), 284-332 (2014)

8. Forbes-Riley, K., Litman, D., Huettner, A., Ward, A.: Dialogue-learning correlations in spoken dialogue tutoring. In: Proceedings of the 2005 Conference on Artificial Intelligence in Education: Supporting Learning Through Intelligent and Socially Informed Technology, pp. 225-232. IOS Press, Amsterdam (2005). http:// dl.acm.org/citation.cfm?id=1562524.1562559

9. Graesser, A.C., Chipman, P., Haynes, B.C., Olney, A.: Autotutor: an intelligent tutoring system with mixed-initiative dialogue. IEEE Trans. Educ. 48, 612-618 (2005)

10. Gupta, S., Walker, M.A., Romano, D.M.: How rude are you?: Evaluating politeness and affect in interaction. In: Paiva, A.C.R., Prada, R., Picard, R.W. (eds.) ACII 2007. LNCS, vol. 4738, pp. 203-217. Springer, Heidelberg (2007). https://doi.org/ 10.1007/978-3-540-74889-2_19

11. Hennessy, S., et al.: Developing a coding scheme for analysing classroom dialogue across educational contexts. Learn. Cult. Soc. Interact. 9, 16-44 (2016). https://doi.org/10.1016/j.lcsi.2015.12.001. http://www.sciencedirect.com/science/ article/pii/S2210656115300507 
12. Katz, S., O'Donnell, G., Kay, H.: An approach to analyzing the role and structure of reflective dialogue. IJAIED 11, 320-343 (2000). https://telearn.archives-ouvertes.fr/hal-00197333. Pilkington, R. (ed.) Part I of the Special Issue on Analysing Educational Dialogue Interaction

13. Maharjan, N., Rus, V.: A tutorial Markov analysis of effective human tutorial sessions. In: Proceedings of the 5th Workshop on Natural Language Processing Techniques for Educational Applications, pp. 30-34. Association for Computational Linguistics, Melbourne, Australia, July 2018. https://doi.org/10.18653/v1/W183704. https://www.aclweb.org/anthology/W18-3704

14. Maharjan, N., Rus, V., Gautam, D.: Discovering effective tutorial strategies in human tutorial sessions. In: The Thirty-First International Flairs Conference (2018)

15. Markus, M.: Politeness in interaction: an analysis of politeness strategies in online learning and teaching (2011)

16. McLaren, B.M., DeLeeuw, K.E., Mayer, R.E.: A politeness effect in learning with web-based intelligent tutors. Int. J. Hum Comput Stud. 69(1-2), 70-79 (2011)

17. McLaren, B.M., Lim, S.J., Yaron, D., Koedinger, K.R.: Can a polite intelligent tutoring system lead to improved learning outside of the lab? Front. Artif. Intell. Appl. 158, 433 (2007)

18. Nashruddin, N.: Politeness principles used by EFL teacher in classroom interaction and its implication toward teaching-Learning Process. Ph.D. thesis, Universitas Negeri Makassar (2017)

19. Pearson, N.K., Kreuz, R.J., Zwaan, R.A., Graesser, A.C.: Pragmatics and pedagogy: conversational rules and politeness strategies may inhibit effective tutoring. Cogn. Instr. 13(2), 161-188 (1995)

20. Rus, V., D’Mello, S., Hu, X., Graesser, A.: Recent advances in conversational intelligent tutoring systems. AI Mag. 34(3), 42-54 (2013)

21. VanLehn, K., Graesser, A.C., Jackson, G.T., Jordan, P.W., Olney, A., Rosé, C.P.: When are tutorial dialogues more effective than reading? Cogn. Sci. 31(1), 3-62 (2007)

22. Wang, N., Johnson, W.L., Mayer, R.E., Rizzo, P., Shaw, E., Collins, H.: The politeness effect: pedagogical agents and learning outcomes. Int. J. Hum.Comput. Stud. 66(2), 98-112 (2008). https://doi.org/10.1016/j.ijhcs.2007.09.003. http://www.sciencedirect.com/science/article/pii/S1071581907001267

23. Wang, N., Johnson, W.L., Rizzo, P., Shaw, E., Mayer, R.E.: Experimental evaluation of polite interaction tactics for pedagogical agents. In: Proceedings of the 10th International Conference on Intelligent User Interfaces, pp. 12-19 (2005)

24. Yao, L.: How polite you are: A study of learners' politeness strategies used in avatars in the second life virtual world (2018)

25. Yoga, W.I., Ketut, S.I., Hery, S.M.: The implications of politeness strategies among teachers and students in the classroom (2018) 